\title{
P04.42. Use of complementary and alternative medicine among adults with neuro-psychiatric symptoms common to mild traumatic brain injury
}

\author{
M Purohit ${ }^{1 *}$, R Wells ${ }^{2}$, S Bertisch ${ }^{1}$, R Zafonte ${ }^{3}$, R Davis $^{1}$, R Phillips $^{1}$ \\ From International Research Congress on Integrative Medicine and Health 2012 \\ Portland, Oregon, USA. 15-18 May 2012
}

\section{Purpose}

One in three adults uses complementary and alternative medicine (CAM) annually in the United States. However, the pattern of CAM use among adults with neuropsychiatric symptoms commonly reported by patients with mild traumatic brain injury (mTBI), a serious public health concern, is not well studied.

\section{Methods}

We analyzed data from the 2007 National Health Interview Survey $(n=23,393)$ to compare CAM use between adults with and without neuropsychiatric symptoms common to mTBI. Symptoms included self-reported anxiety, depression, insomnia, headaches, memory deficits, attentional deficits, and excessive sleepiness. CAM use was defined as use of mind-body (e.g., meditation), biological (e.g., herbs), manipulation (e.g., massage) therapies, and alternative medical systems (e.g., Ayurveda), within the past 12 months. We estimated prevalence and reasons for CAM use in patients with and without neuropsychiatric symptoms. We also explored variations in CAM use by the number of symptoms. Multivariable logistic regression was performed to examine the association between neuropsychiatric symptoms and CAM use after adjustment for sociodemographic characteristics, illness burden (e.g,. fibromyalgia, low back pain), access to care, and health habits.

\section{Results}

Adults with neuropsychiatric symptoms had higher CAM use compared to adults without neuropsychiatric symptoms $(44 \%$ vs. $30 \%, \mathrm{p}<0.001)$; prevalence increased with
Table 1

\begin{tabular}{cccc}
\hline Number of Symptoms & aOR & \multicolumn{2}{c}{$\mathbf{9 5 \%} \mathbf{C l}$} \\
\hline 0 & 1.00 & & \\
1 & 1.43 & 1.32 & 1.56 \\
2 & 1.70 & 1.51 & 1.91 \\
$\geq 3$ & 1.77 & 1.56 & 2.01 \\
\hline
\end{tabular}

increasing number of symptoms ( $\mathrm{p}$-value for trend $<0.001$, table below). Differences persisted after adjustment (table below). Twenty percent used CAM because standard treatments were either too expensive or ineffective; $25 \%$ used CAM because it was recommended by a provider.

\section{Conclusion}

More than $40 \%$ of adults with neuropsychiatric symptoms observed in mTBI used CAM. An increasing number of symptoms was associated with increased use. Future research is needed to understand the use, efficacy, and safety of CAM in mTBI patients.

\section{Author details}

${ }^{1}$ Beth Israel Deaconess Medical Center/Harvard Medical School, Boston, USA. 2Brigham and Women's Hospital/Faulkner Hospital/Harvard Medical School, Boston, USA. ${ }^{3}$ Spaulding Rehabilitation Hospital/Harvard Medical School, Boston, USA.

Published: 12 June 2012

doi:10.1186/1472-6882-12-S1-P312

Cite this article as: Purohit et al: P04.42. Use of complementary and alternative medicine among adults with neuro-psychiatric symptoms common to mild traumatic brain injury. BMC Complementary and Alternative Medicine 2012 12(Suppl 1):P312. 